\title{
Long-term positive and negative identity priming: Evidence for episodic retrieval
}

\author{
DOUG LOWE \\ Trent University, Peterborough, Ontario, Canada
}

\begin{abstract}
An episodic retrieval account of negative priming (Neill, 1997; Neill \& Valdes, 1992) was evaluated in three experiments. During practice, regular word pairs were presented to subjects differing numbers of times. The subjects named specific target words while they ignored specific distractor words. Following a 5-min retention interval, memory for practice was revealed: Test responses for target words exhibited positive priming that increased with increases in the number of times that the words had been attended. Test responses for distractor words exhibited either positive priming (Experiment 1) or negative priming (Experiments 2-3) that also increased with increases in the number of times that the words had been ignored. The type of priming that distractors exhibited was determined by several contextual similarities between the practice environment, in which distractors were ignored initially, and the test environments, in which they were processed subsequently. Negative priming that spanned a 5-min interval, increased with increases in the number of times that a distractor was ignored, and was sensitive to contextual changes indicated that the direction of the effect was temporally backward because the test probe cued memory for earlier processing of the priming stimulus when the distractor had been ignored.
\end{abstract}

The deployment of selective attention permits subjects to respond optimally to target aspects of stimulation while ignoring distractor aspects. There is now substantial evidence that selecting for, or facilitation of, target information entails selecting against, or inhibition of, distractor information. Distractor inhibition has been inferred from the findings of priming procedures during which the distractor item for a leading, or priming, stimulus becomes the target item for a trailing, or probe, stimulus. According to the logic of the procedure, ignoring an item by means of its inhibition on the prime trial will make the same item difficult to process on the subsequent probe trial.

Dalrymple-Alford and Budayr (1966) first demonstrated a distractor inhibition effect in a list of Stroop (1935) color words. When each distractor color word in the list named the next ink color, color-naming responses were slower and less accurate than when adjacent color words were unrelated. Neill (1977) confirmed the finding in a discrete trials version of the task, and made the important interpretation that the distractor representations for the prime had been selectively inhibited. To contrast the processing impairment for ignored stimuli with the facilitated processing, or positive priming (PP), produced for attended stimuli, Tipper

This research was conducted from 1986 to 1989 while the author was funded by the National Science and Engineering Research Council of Canada (Grant AO 284). I am indebted to Mike Masson, Bruce Milliken, Tramm Neill, and Bruce Whittlesea for many insightful comments on an earlier version of this manuscript. Correspondence should be addressed to D. Lowe, Department of Psychology, Trent University, Peterborough, ON, Canada K9J 7B8 (e-mail: dlowe@trentu.ca).

-Accepted by previous editor, Geoffrey R. Loftus
(1985) termed transfer effects of this type negative priming (NP). Subsequent research has shown the wide applicability of NP to a broad range of stimuli and tasks (see Fox, 1995; Houghton \& Tipper, 1994; May, Kane, \& Hasher, 1995; Neill, Valdes, \& Terry, 1995, for reviews).

In considering the meaning of NP, Neill (1977) speculated that inhibition of distractor representations resulted in reduction of their activation below base levels. The "deactivated" representations lingered into the probe trial, and performance was impaired because the appropriate responses were temporarily unavailable. Neumann and DeSchepper (1991) proposed that inhibition resulted in a raised threshold of activation for distractor representations. Tipper and Cranston (1985) subsequently modified Neill's (1977) proposal by arguing that inhibited representations remained activated, but were "blocked" from access to response mechanisms. However, to come full circle, Houghton and Tipper (1994) proposed that, at prime offset, there are negative levels of activation for distractor representations. Clearly, there are shifting opinions on the meaning of NP.

Yet despite this absence of a theoretical consensus, the different accounts agree that the direction of NP effects is temporally "forward" from the prime, via lingering persistence of a weakened distractor representation, into the probe trial. Recent findings, however, suggest that NP may neither index selective processing of the prime, nor reflect active inhibition of the distractor.

While investigating the duration of NP, Neill and his colleagues (Neill \& Valdes, 1992; Neill, Valdes, Terry, \& Gorfein, 1992) discovered that although NP can last up to several seconds, the dissipation of NP was greatly reduced when the temporal interval between the response to the prime and the onset of the probe matched the interval that preceded 
the prime. In contrast, when the intervals surrounding the prime were not constant, greater dissipation of NP was observed (see also Hasher, Zacks, Stolzfus, Kane, \& Connelly, 1996; Neill, 1997). Since events preceding the prime affect the duration of NP, it cannot be assumed that probe performance is a direct index of selective processing of the prime or the activation state of distractor representations. Moreover, the observation that NP is affected by events that surround the prime and possibly by events that surround the probe suggests that NP may be determined by broader types of prime-probe relationships beyond those involving prime distractors and probe targets.

To account for their findings, Neill (Neill \& Valdes, 1992; Neill et al., 1992) attributed NP to memory retrieval of the priming episode, during which the probe target was ignored. Baddeley's (1976) proposal that the success of retrieving an epısode depends on its temporal discriminability from other episodic memories explains the near identity of NP at constant short and long intervals. When applied to the NP task, temporal discriminability of the priming episode would depend on the ratio of the most recent primeprobe delay to the delay between the prime and previous trials. When the current prime-probe delay is short, and the interval before the prime is long, the probability of retrieving the priming episode is high, yielding NP. Conversely, when the current prime-probe interval is long, and the delay before the prime is short, the probability of retrieving the priming episode is low, yielding reduced NP. When the intervals surrounding the prime are constant, despite their durations, the ratio is the same. Consequently, there is equal discriminability of the priming episodes, and retrieval probability is unaffected, yielding equal degrees of NP. More recently, Neill (1997) has directly shown the importance of temporal discriminability for retrieval of the priming episode, and for NP. By manipulating the target-distractor onset interval for both prime and probe trials, it was shown that NP was dependent on the identity of distractor onset conditions between primes and probes. When primes and probes did not share the same distractor onset information, retrieval did not occur, and NP was not obtained.

The processes implicated by a retrieval account of NP are similar to those posited in Logan's (1988) instance theory of automaticity. According to the theory, performance of any cognitive task is initially accomplished by an algorithmic computation. Each time the task is performed, a separate episodic trace, or instance, is stored in memory. The episode contains information about a particular stimulus event and all information that was associated with it in the past, including the response that was made. Attributes important for performing the task are attended and are incorporated into the episode; presumably, in the NP task, attributes for the prime target are coded as "to-be-attended," or "reportable." Subsequent encounters with the stimulus cue memory retrieval of the priming episode, providing the correct response. With repetition of the task, more instances accumulate in memory, and correct responses are retrieved from memory before they can be provided by the algorithm.

To apply the theory to NP, Neill and Valdes (1992) argued that information about ignored events is also encoded into memory; presumably, in the NP task, attributes for the prime distractor are coded as "to-be-ignored" or "nonreportable." Presentation of ignored items as probe targets cues retrieval of the priming episodes, and performance is impaired because of interference between the available "response" and the retrieved "nonresponse" attributes that are concurrently associated with the target.

Alternatively, the distractor having been ignored, it may be that no response information for the distractor gets stored, and responses may be delayed because there is no appropriate response information available. By this account, NP depends on the differential processing of targets and distractors during selective attention to the prime. However, in contrast to the logic of the priming procedure, the direction of NP is temporally "backward" when the probe cues retrieval of memory for past processing of the distractor. Accordingly, the processing impairments during NP trials do not reflect distractor inhibition during selective attention to the prime.

Despite its apparent promise, Logan's (1988) theory may be problematic when applied to the NP task. Typical NP studies use a few stimuli that are balanced to serve equally as targets and distractors and that are presented repeatedly throughout the experiment. Malley and Strayer (1995) have shown that NP is stimulus specific, restricted to highly familiar stimuli experienced before. They found that NP occurred only when an ignored distractor was a stimulus that had been repeated often throughout the experiment. PP, and not NP, occurred when a distractor was novel and had not been experienced before the prime.

Thus, when the same stimuli are used repeatedly throughout the experiment, in principle, on any given NP probe trial, a retrieved episode could contain the "reportable" attribute as often as it contained the "nonreportable" attribute. In such an unstable environment, memory for past processing would provide little useful information (Logan, 1988). Therefore, how retrieval of past episodes would affect performance is not transparent, even if that retrieval was certain to occur. Indeed, when "reportable" and "nonreportable" attributes are equally retrievable, it could be predicted that the attributes might cancel, and there would be no NP at all.

Experiment 1 was a direct test of a memory-based account of NP. To avoid problems inherent in the same stimuli's serving as both targets and distractors, individual words consistently served as either targets or distractors, but not both. The words were painted in different ink colors, and, on each trial, subjects attended, and named aloud, the word in the target color while they ignored the word in the nontarget color. Following Logan (1988), during practice specific word pairs were presented one, three, six, or nine times. Of necessity, repetition of the items caused the final presentations of the most presented pairs to occur during 
the latter part of practice. To eliminate any "recency advantage" for these pairs, there was an unfilled 5-min retention interval before test.

Following the retention interval, a test phase involved presentations of probe stimuli that were designed to yield single, one time, reaction time (RT) measures of both the type and degree of priming for target and distractor words from practice. One third of the test trials assessed $\mathrm{PP}$ for targets, which had been attended one, three, six, or nine times in an attended repetition (ATT) condition (Tipper, 1985 ) that involved a final presentation of prime word pairs from practice. Another third of the test trials assessed NP for distractors, which had been ignored one, three, six, or nine times in an ignored repetition (IGN) condition (Tipper, 1985). As in a typical IGN condition, distractor words from practice were painted in the target color. However, unlike in a typical IGN condition, to ensure the consistency of targets and distractors, test (i.e., probe) targets were paired with novel words that were not part of practice. The novel words served as the probe distractors and were painted in the nontarget color. On the remaining test trials, which provided the baseline for assessing the degree of priming for ATT and IGN trials, novel (new) word pairs were presented that were unrelated to practice.

Memory-based theories (Logan, 1988; Logan \& Etherton, 1994) propose that practice at attending and reporting target members of the pairs should create episodic memories that contain the attributes that specific words are consistently "reportable." With repetitions, more traces of the pairs and the appropriate responses should accumulate in memory. Subsequent presentations of the pairs during ATT test trials should cue retrieval of that knowledge, yielding progressively improved performance, or PP, with increases in number of repetitions. According to Neill and Valdes (1992), ignoring distractor members of the repeated pairs should create episodic memories that also include the knowledge that specific words are consistently "nonreportable," or memories that lack response information. Subsequent presentations of the distractors as targets during IGN test probes should cue retrieval of those episodes, yielding progressively impaired performance, or $\mathrm{NP}$, with increases in the number of presentations. "Longterm" NP that spans an interval of $5 \mathrm{~min}$ and that increases with the number of times that an item has been ignored would implicate memory retrieval as the cause of the processing impairment. Neuman and DeSchepper (1992) have shown NP effects in a short-term memory task, and there is evidence from a shape-matching task (DeSchepper \& Treisman, 1991, 1996) that NP can be long term, and can increase with increases in the number of times that a distractor is ignored (but see Malley \& Strayer, 1995, p. 658).

To anticipate the findings, in different experiments, test responses for distractors exhibited either PP or NP. In Experiment 1 , both targets and distractors exhibited progressive PP with increases in the number of presentations. It appeared that between the practice and test phases, subjects may have detected a contextual change that was caused by unexpected new pairs during test. Experiment 2 was a test of this possibility; new items were eliminated, and the test trials were composed entirely of ATT and IGN items. Under these circumstances, distractors exhibited progressive NP with increases in the number of presentations. Experiment 3 was a study of a second contextual determinant of NP that emerged in Experiments 1 and 2.

\section{EXPERIMENTS 1 AND 2}

\section{Method}

Design and Materials. One hundred ninety-two words, three to five letters in length, were selected from clusters seven and eight of the Battig and Togglia (1978) word norms. One hundred forty-four of the words were randomly selected to create 72 pairs while ensuring that the items were not synonyms, did not rhyme, and had no obvious association (e.g., BOX and CAR). For each pair, the words were printed above one another on white cards in uppercase 42-point Letraset Instant Lettering (No. 723). For one half of the pairs, the upper word was painted in red ink and the lower word was presented in blue. For the remaining pairs, the colors were reversed.

Forty-eight pairs were randomly selected to serve as practice stimuli; the remaining 24 pairs served as new test items. The 48 practice pairs were also altered to serve as IGN probes by replacing target words with 1 of the remaining 48 words that had been selected from the norms. The words that served as prime distractors remained in their same upper or lower position but appeared in the target color. The replacement (i.e., probe distractor) word was painted in the nontarget color. For example, the pair BOoK in red above FISH in blue was altered to DRUM in blue above FISH in red. For a subject naming red words, FISH would be the prime distractor and probe target, respectively. A second set of IGN stimuli was similarly constructed by replacing the other word (i.e., FISH). Overall, there were 120 different pairs, and each was photographed separately onto $35-\mathrm{mm}$ slides.

Both experiments involved 300 trials composed of 228 practice trials followed by 72 test trials, with a 5 -min interval between. The practice phases of all experiments were identical. Two different presentation orders of 144 trials each were used, with one half of the subjects each receiving a different order. The subjects were presented the 48 word pairs, which had been randomly separated into four sets of 12 pairs each. Enough examples were created so that a particular set could be presented one, three, six, or nine times. The stimuli for the four presentation conditions were fairly evenly distributed throughout practice, with the restriction that no identical pair or position of the same colored item could appear more than twice in succession. One half of the subjects named red words, and the remainder named blue words. For one half of the subjects, the practice pairs that served as ATT stimuli during test were used as IGN probes for the other half of the subjects. For the remainder of the subjects, this relationship was reversed.

Within the test phase of each experiment, all subjects received the identical stimulus order. There were 24 new items and 6 pairs for each number of practice presentations for both ATT and IGN conditions. In Experiment 1, the 24 pairs for each ATT, new, and IGN test condition were randomly intermixed with the same restrictions used for practice. In Experiment 2, there were no new test items; the 24 stimuli within each ATT and IGN test condition appeared in the same order as in Experiment 1.

Procedure. All stimuli were back projected onto an opaque screen via a Kodak carousel projector that was fitted with a Gerbrands shutter. When the displays were viewed from a distance of approximately $66 \mathrm{~cm}$, their subtended visual angles varied from approximately $3.3^{\circ}$ $\times 2.2^{\circ}$ for three-letter words to $5.5^{\circ} \times 2.2^{\circ}$ for five-letter words. The separation between upper and lower words was approximately $2.2^{\circ}$. 
Table 1

Mean Reaction Times (in Milliseconds),

With Standard Deviations, for Practice Trials

as a Function of Number of Practice

Presentations (Experiments 1-3)

\begin{tabular}{|c|c|c|c|c|c|c|c|c|c|c|c|c|}
\hline \multirow[b]{3}{*}{ Experiment } & \multicolumn{12}{|c|}{ No. of Practice Presentations } \\
\hline & \multicolumn{2}{|c|}{1} & \multicolumn{2}{|c|}{2} & \multicolumn{2}{|c|}{3} & \multicolumn{2}{|c|}{$4 / 5$} & \multicolumn{2}{|c|}{$6 / 7$} & \multicolumn{2}{|c|}{$8 / 9$} \\
\hline & $M$ & $S D$ & $M$ & $S D$ & $M$ & $\overline{S D}$ & $M$ & $S D$ & $M$ & $S D$ & $M$ & $S D$ \\
\hline 1 & 673 & 66.4 & 634 & 65.4 & 628 & 67.2 & 617 & 65.8 & 619 & 76.0 & 606 & 76 \\
\hline 2 & 660 & 58.4 & 619 & 52.6 & 614 & 59.1 & 610 & 66.2 & 608 & 63.5 & 597 & $6 ?$ \\
\hline 3 & 658 & 56.7 & 618 & 52.0 & 613 & 55.1 & 611 & 58.7 & 609 & 62.6 & 586 & 69.5 \\
\hline
\end{tabular}

Presentation times were controlied by an Apple II+ microprocessor, which also measured and collected RTs.

Each trial began with a central fixation dot on the blank screen, above and below which the words appeared. The subjects initiated stimulus presentation, which simultaneously started a software timer. The responses, registered with a voice key, simultaneously (1) stopped the timer, (2) removed the stimulus from view and (3) advanced the projector, which signaled the onset of the next trial. The intertrial interval was approximately $3 \mathrm{sec}$. The subjects were instructed (1) to name the word that was painted in the target color as quickly and accurately as possible, (2) that, although from trial to trial the location of the target word was unpredictable, the word would appear in both locations equally often, and (3) that some pairs would appear more than once. Between practice and test trials, the subjects chatted with the experimenter.

Subjects. The 40 subjects in each experiment were male and female undergraduates at Trent University who participated as a course requirement.

\section{Results and Discussion}

The rejection region for all statistical tests was $p<.01$, or better, unless stated otherwise. Data from trials on which subjects' responses were incorrect or on which subjects failed to stop the clock were excluded from analysis. The error data were too few to allow meaningful analyses; only 5 and 6 subjects in Experiments 1 and 2, respectively, made any errors.

Practice trials: Experiments 1 and 2. For each subject, median RTs were calculated for each number of presentations. Since items that were repeated three, six, or nine times contributed practice data to all earlier presentations, medians for one presentation were based on the presentation of all 48 items. For two and three presentations, medians were based on the 36 pairs that received three, six, or nine presentations. For four through nine presentations, medians were based on the 24 pairs that received either six or nine presentations. However, before calculating the medians, data for four and five presentations, six and seven presentations, and eight and nine presentations were combined. Thus, medians for one, two, three, four and five, six and seven, and eight and nine presentations were based on $48,36,36,48,36$, and 24 , or fewer, correct RTs, respectively. The means of subjects' median RTs, for each number of presentations, are shown in Table 1.

Across experiments, the pattern of results was virtually identical. One-way repeated measures ANOVAs showed that average RTs decreased as the number of presentations increased [Experiment $1, F(5,195)=37.06, M S_{\mathrm{e}}=563$; Experiment 2, $\left.F(5,195)=35.53, M S_{\mathrm{e}}=544\right]$. The greatest improvements occurred between one and two presentations. Except for one minor reversal between four and five presentations and six and seven presentations in Experiment 1 , in both experiments, there were small, progressive, RT improvements following two presentations. Additional ANOVAs, that excluded one-presentation data, showed that RTs decreased between two presentations and eight and nine presentations [Experiment $1, F(4,156)=9.59$, $M S_{\mathrm{e}}=490$; Experiment $\left.2, F(4,156)=5.58, M S_{\mathrm{e}}=505\right]$.

Progressive RT improvements, or PP, with increasing numbers of presentations show that practice at reporting specific targets for the repeated pairs progressively aided subsequent performance. Presumably, subjects learned and remembered which words were "reportable." Subsequent encounters with the pairs cued retrieval of that knowledge, facilitating performance, as instance theory (Logan, 1988) predicts.

The greater improvement between one presentation and two than between subsequent repetitions shows that there were "warmup effects," so that performance was affected also by general or nonspecific practice (Logan, 1988). General practice would increase with progressive trials, so the RT improvements could have been due entirely to nonspecific practice. To gauge the extent of nonspecific practice, data for pairs that were presented only once, and for which specific practice was impossible, were categorized according to whether they occurred during the first or second half of practice. For each subject, median RTs were calculated.

In both experiments, mean RTs for nonrepeated items from the second half of practice were faster than during the first half, showing the presence of nonspecific practice [Experiment 1, first half $M=683 \mathrm{msec}$ vs. second half $M=$ $657 \mathrm{msec}, t(39)=3.88$; Experiment 2 , first half $M=$ $668 \mathrm{msec}$ vs. second half $M=646 \mathrm{msec}, t(39)=3.21$ ]. If PP for repeated pairs was due entirely to nonspecific practice, performance for repeated and nonrepeated items should have been nearly identical across practice. However, during the second half, RTs for repeated pairs (i.e., four and five, six and seven, and eight nine presentations combined) were substantially faster than RTs for nonrepeated items [Experiment 1, nonrepeated $M=657 \mathrm{msec}$ vs. repeated $M=615 \mathrm{msec}, t(39)=5.93$; Experiment 2, nonrepeated $M=646 \mathrm{msec}$ vs. repeated $M=605 \mathrm{msec}$, 
Table 2

Mean Reaction Times (in Milliseconds), With Standard Deviations, for Test Trials as a Function of Number of Practice Presentations and Test Response Condition (Experiments 1-3)

\begin{tabular}{|c|c|c|c|c|c|c|c|c|c|c|}
\hline \multirow[b]{4}{*}{ Experiment } & \multicolumn{10}{|c|}{ Response Condition } \\
\hline & \multicolumn{4}{|c|}{$\begin{array}{l}\text { Attended } \\
\text { Repetition }\end{array}$} & \multirow{2}{*}{\multicolumn{2}{|c|}{$\begin{array}{c}\text { Control } \\
0\end{array}$}} & \multicolumn{4}{|c|}{$\begin{array}{c}\text { Ignored } \\
\text { Repetition }\end{array}$} \\
\hline & \multicolumn{2}{|c|}{$1 / 3$} & \multicolumn{2}{|c|}{$6 / 9$} & & & \multicolumn{2}{|c|}{$1 / 3$} & \multicolumn{2}{|c|}{$6 / 9$} \\
\hline & $M$ & $S D$ & $M$ & $S D$ & $M$ & $S D$ & $M$ & $S D$ & $M$ & $S D$ \\
\hline 1 & 654 & 84.7 & 636 & 78.0 & 675 & 72.5 & 658 & 81.9 & 641 & 80.6 \\
\hline 2 & 624 & 69.5 & 610 & 55.3 & & & 639 & 71.2 & 656 & 92.9 \\
\hline 3 & 636 & 68.3 & 621 & 70.1 & 641 & 67.9 & 642 & 79.1 & 664 & 69.0 \\
\hline
\end{tabular}

$t(39)=8.09]$. The advantage for repeated pairs is consistent with the idea that learning was item specific, and that performance was instance or item based.

Test trials: Experiment 1. Since there were only six scores for each ATT and IGN condition for each number of practice presentations, the data were quite variable. Consequently, data for trials with one and three practice presentations and with six and nine practice presentations were combined before calculating the medians. It should be noted that distractors for ATT probes were the same during practice and test, whereas IGN test phase distractors were new words, so that a direct comparison between these items may not be meaningful. More interpretable results are revealed by the effects of number of practice presentations on ATT and IGN targets. Mean RTs are presented in Table 2.

The design was not a complete factorial, so several analyses were used. To evaluate response conditions, data for each subject were collapsed across number of practice presentations (i.e., one, three, six, nine), and medians were calculated for each ATT, new, and IGN test condition. A one-way repeated measures ANOVA revealed that RTs differed as a function of conditions $\left[F(2,78)=4.44, M S_{\mathrm{e}}=\right.$ 437]. RTs for ATT ( $M=645 \mathrm{msec})$ and IGN $(M=649 \mathrm{msec})$ trials did not differ (see also next analysis), but both were faster than responses for new trials.

A $2 \times 2$ repeated measures ANOVA was used to evaluate number of practice presentations and ATT versus IGN trials; RTs for ATT and IGN trials did not differ $[F(1,39)$ $\left.<1, M S_{\mathrm{e}}=3,534\right]$, nor did response condition interact with number of presentations $\left[F(1,39)<1, M S_{\mathrm{e}}=3,047\right]$. Overall RTs decreased with number of practice presentations, although the effect was marginally reliable $[F(1,39)=$ $2.92, M S_{\mathrm{e}}=4,324, p<.10$; see next analysis].

The effect of number of practice presentations was examined in a third analysis by collapsing across ATT and IGN trials. For each subject, median RTs for each number (i.e., zero [new], one/three, six/nine) of presentations were calculated. A one-way repeated measures ANOVA showed that RTs decreased significantly with increases in number of presentations $\left[F(2,78)=7.30, M S_{\mathrm{e}}=532\right]$. Using the $M S_{\mathrm{e}}$ from the ANOVA as the estimate of error, subsequent $t$ tests showed that RTs for trials with six/nine presentations $(M=639 \mathrm{msec})$ were faster than those for trials with one/three presentations $[M=656 \mathrm{msec} ; t(39)=3.24]$, which in turn were faster than RTs for new items [ $M=$ $675 \mathrm{msec}, t(39)=4.02]$.

Progressive PP with number of repetitions for both target and distractor words indicates that test performance was memory based. The specific activities of attending particular target words while ignoring particular distractor words were not differentially influential, but frequency of exposure to both item types was. PP for IGN trials could be an embarrassment for the inhibitory theory; apparently, the representations of target and distractor words were equally available during test. PP for distractor processing might also contradict Neill and Valdes's (1992) notion that information that distractors were "nonreportable" was included, or that appropriate response information was lacking, in the retrieved episodes.

However, these results were obtained with procedures quite different from those usually used to test for NP (i.e., a large set of words as stimuli, consistency of stimuli as targets and distractors, multiple practice on items, novel items as control stimuli, novel items as probe distractors, long-term test, etc.). Any one of these changes might have eliminated NP. During practice, for example, the distractors might not have been ignored; with repetitions, subjects might have deliberately attended to them. Perhaps IGN trials failed to exhibit NP because distractor inhibition never took place (i.e., if NP had been measured during practice). Experiment 2 was performed to address these issues.

Tipper, Weaver, Cameron, Brehaut, and Bastedo (1991) found that NP was reduced when novel, unexpected, events intervened between primes and probes. Their test trials included unexpected presentations of new pairs that were unrelated to practice. Given that their subjects expected repeated word pairings during practice, the introduction of unfamiliar new items might have signaled that there had been a contextual change, thereby eliminating NP (Tipper et al., 1991). In the present Experiment 2, elimination of new word pairs might cause practice and test phases to appear more alike, thereby yielding NP.

Test trials: Experiment 2. Since there were no new probes, a sole $2 \times 2$ repeated measures ANOVA was used to evaluate the effects of number of practice presentations on ATT versus IGN trials. Unlike in Experiment 1, the test responses for distractors were slower than those for targets $\left[F(1,39)=19.09, M S_{\mathrm{e}}=1,949\right]$, and response condition interacted with number of presentations $[F(1,39)=$ $\left.11.14, M S_{\mathrm{e}}=922\right]$. Using the $M S_{\mathrm{e}}$ from the ANOVA as the estimate of error, subsequent $t$ tests showed that RTs for ATT trials decreased with increases in the number of presentations $[t(39)=2.19, p<.05]$, while RTs for IGN trials increased $[t(39)=2.57, p<.05]$.

The presence of NP for IGN targets shows that distractor words were ignored during practice, and that subjects did not deliberately attend to them. Progressive PP and NP for targets and distractors, respectively, also suggests that test performance was memory based. Knowledge that target words were "reportable" was stored in the practice episodes that were retrieved during ATT trials (Logan, 1988). More 
important, knowledge that distractors were "nonreportable" was also stored in the practice episodes that were retrieved during IGN trials (Neill \& Valdes, 1992).

NP that spans an interval of 5 min clearly implicates memory retrieval of past processing as the source of the processing impairment. It may be that distractors were "selected against" when they were ignored during practice (but see Milliken \& Joordens, 1996, and Milliken, Joordens, Merikle, \& Seiffert, 1995, for evidence that prime selection may not be necessary for NP), but it is not possible that distractor representations were "deactivated" (Houghton \& Tipper, 1994; Neill, 1977; Neumann \& DeSchepper, 1991) or "blocked" (Tipper, 1985). If NP were caused by persistence of inhibited distractor representations into test, it is unlikely that they would be rendered unavailable for such an extended duration. When considered in isolation from Experiment 1, the findings provide considerable support for Neill and Valdes's (1992) retrieval account of NP.

The pattern of results reflects context-specific learning during practice. NP is sensitive to broader types of primeprobe relationships than those involving past distractors and current targets. Targets and distractors for the repeated pairs became connected to the environment or general background in which those items were processed. Apparently, general context was used along with specific test probes during memory access, for what was retrieved depended on whether or not the "list-wide" context from practice was reinstated during test. When the learning (i.e., practice) and retrieval (i.e., test) contexts were consistent, distractor processing was impaired; when those contexts were inconsistent, distractor processing was facilitated. The contexts would have been consistent when there were no new items, because test responses consisted entirely of the contents of practice episodes. Contextual inconsistency did not prevent the influence of earlier episodes; rather, it changed the way that processing was influenced. Memory for the specific activities of ignoring particular distractor words was context dependent, whereas memory for frequency of exposure to them was context independent. Overall, the results have something in common with other reports of "list-wide" context effects on PP during word recognition tasks (e.g., Chawarski \& Sternberg, 1993; Neely \& Durgunoğlu, 1985; Smith, Macleod, Bain, \& Hoppe, 1989 ), suggesting that a common mechanism may underlie PP and NP.

Context dependency of NP strongly reinforces the claim that the direction of the different priming effects was temporally "backward" when the test probes cued memory for past processing. According to "forward" accounts of NP (e.g., Malley \& Strayer, 1995; Neill, 1977; Tipper \& Cranston, 1985), impaired performance for ignored stimuli should be "short term" and should be conditional only on how the distractors have been processed initially; it should not be "long term" and dependent on the context in which they are processed subsequently.

Context dependency of NP also indicates that the results have several commonalities with more typical demonstrations of NP (e.g., a small set of stimuli, stimuli serving inconsistently as targets and distractors, control items selected from the repeating stimulus set, short-term test, etc.). Lowe (1979; Experiment 4) documented that whether particular probes exhibited PP or NP was controlled by the context that was set by the "mix" of different types of probes that were used within an experiment; similar findings have been reported by Moore (1994). As noted, Tipper et al. (1991) showed that NP is decreased when the test context includes unfamiliar events. Lowe (1979; Experiment 2) showed that NP was more likely to occur when there were many repeated target (i.e., ATT) trials between primes and probes, so that NP can be contextually dependent on subjects' strategies to use information taken from primes to process probes. In Experiment 1, ATT items made up one third of the test items, so the small proportion of repeated trials may have discouraged subjects' use of memory for practice pairs for responding to test items, thereby eliminating NP. In Experiment 2, ATT items made up one half of the test trials, thus increasing the likelihood that memory for practice items would be useful for processing test stimuli, thereby yielding NP.

However, practice-test contextual dissimilarity in Experiment 1 cannot explain why IGN test probes displayed PP. If contextual changes prevented retrieval of processing episodes from training, RTs for IGN and new trials should have been similar. Typical studies of NP have also shown that NP sometimes reverses to PP when probes consist of nonconflict stimuli (e.g., Lowe, 1979; Tipper \& Cranston, 1985). Neill (1997) has suggested that contextual dissimilarity between conflicting prime stimuli followed by nonconflicting probes prevents episodic retrieval, thereby eliminating NP. To account for PP for nonconflict probes, Neill (1997) proposed that there may be a second source of PP that is independent of contextual similarity-that is, activation may persist at some lower level of representation. Thus, whereas NP depends on retrieval context, PP for distractor processing may result in part from a different mechanism that is insensitive to context, such as persistent activation in a semantic memory system.

\section{EXPERIMENT 3}

When distractors became test targets, they were paired with novel words, so they appeared in different contexts than when they were ignored. If contextual consistency is critical for NP, it might have been the case that NP failed to occur at all. Thus, unlike in earlier studies (e.g., Lowe, 1979; Malley \& Strayer, 1995; Moore, 1994), in the present paradigm, composition of the specific test probes might be unimportant for NP. In Experiment 3, test responses for distractors were studied when the items appeared in the same specific context as when they were ignored during practice. In this experiment, IGN probes consisted of the same word pairs that were used during practice. The words appeared in the same upper and lower locations, but their colors were "flipped" so that the distractors became targets and vice versa. "Flipped" probes have not been commonly used in NP experiments, presumably because of the complications 
of simultaneously reversing the roles of both targets and distractors between primes and probes. However, using the Stroop task, Lowe (1979; Experiment 1) documented that "flipped" probes do exhibit NP. As in earlier experiments, individual words consistently served as either targets or distractors. Target-distractor consistency was preserved when the pairs were repeated during ATT probes; that consistency was reversed during "flipped" IGN probes.

According to Tulving and Thomson's (1973) encoding specificity principle, reinstated encoding contexts provide better retrieval cues because the practice episodes closely resemble the test items that have cued them. Therefore, when distractors appear at test in the same specific context in which they were ignored during practice, the more that responses can be made by retrieval alone, and the more that performance should be affected by memory for earlier processing. Thus, "flipped" probes may reveal NP despite the contextual changes that accompany the introduction of new items.

\section{Method}

General. Except for the makeup of IGN probes, in all other aspects Experiment 3 was identical to Experiment 1.

Subjects. The 40 new subjects were selected from the same population as were the subjects in the earlier experiments.

\section{Results and Discussion}

RT data were scored and analyzed as in Experiment 1. Since only 4 subjects made any errors, errors were too few to allow analyses.

Practice trials. Table 1 shows that, as before, RTs decreased reliably with increases in number of presentations $\left[F(5,195)=34.21, M S_{\mathrm{e}}=458\right]$. The greatest $\mathrm{RT}$ reductions were between one and two presentations, and there were small, progressive, improvements between two presentations and eight and nine presentations $[F(4,156)=14.93$, $\left.M S_{\mathrm{e}}=446\right]$.

RTs for nonrepeated items during the second half of practice were reliably faster than those during the first half, indicating nonspecific practice [first half $M=664 \mathrm{msec}$ vs. second half $M=637 \mathrm{msec}, t(39)=3.52]$. However, during the second half, RTs were faster for repeated pairs than for nonrepeated items, indicating that performance for repeated pairs was memory based [nonrepeated $M=$ $637 \mathrm{msec}$ vs. repeated $M=607 \mathrm{msec}, t(39)=7.65$ ].

Test trials. As in Experiment 2, test responses for targets exhibited PP, whereas responses for distractors displayed NP. RTs for ATT $(M=628 \mathrm{msec})$, IGN $(M=$ $658 \mathrm{msec})$, and new probes differed reliably $[F(2,78)=$ $\left.11.96, M S_{\mathrm{e}}=1,082\right]$. Compared with performance for new trials, responses for ATT trials were marginally faster $[t(39)=1.85, p<.10]$, whereas RTs for IGN trials were reliably slower $[t(39)=2.31, p<.05$; see also next analysis $]$.

The $2 \times 2$ repeated measures ANOVA of number of practice presentations and ATT versus IGN response conditions revealed that target responses were faster than distractor responses $\left[F(1,39)=12.63, M S_{\mathrm{e}}=1,824\right]$, and that response condition interacted with number of presentations
$\left[F(1,39)=47.34, M S_{\mathrm{e}}=618\right]$. From Table 2, it is evident that RTs for ATT trials decreased with increases in number of presentations $[t(39)=2.70]$, whereas those for IGN trials increased $[t(39)=3.60]$. Of less interest, overall RTs were unaffected by number of presentations $[F(1,39)<1$, $\left.M S_{\mathrm{e}}=618\right]$. The final ANOVA also indicated that RTs for trials with zero/new $(M=641 \mathrm{msec})$ versus one/three $(M=$ $638 \mathrm{msec})$ versus six $/$ nine $(M=643 \mathrm{msec})$ prior presentations did not differ $\left[F(2,78)<1, M S_{\mathrm{e}}=623\right]$.

Since NP was obtained under conditions where PP was observed earlier, the opposite priming effects for distractors in Experiments 1 and 3 reveal another contextual influence. Presumably, "flipped" items cued retrieval more effectively than the changed probes of Experiment 1 . Test responses for distractors were affected by memory that they had been ignored, yielding NP.

The results bolster earlier claims of the importance of contextual consistency for NP. When distractors became targets in the specific context in which they were ignored, their processing was impaired, despite changes in the general context in which selective processing took place. In Experiment 2, when distractors became targets in a different specific context than when they were ignored, their processing was impaired, but only if the general context in which they had been ignored was maintained. In Experiment 1, when both specific and general contexts changed, distractor processing was facilitated.

\section{GENERAL DISCUSSION}

In three experiments performed to evaluate the claim that NP is a matter of memory retrieval (Neill \& Valdes, 1992), it was demonstrated that NP can be both "long term" and dependent on the number of times that distractors have been ignored. Whether distractor processing exhibited NP or PP depended on several contextual relationships between the sequential environments in which distractors were ignored initially and attended subsequently. NP that is conditional on relationships between the initial processing environment and later test circumstances is contrary to the idea that NP reflects temporary inhibition of distractor information. Rather, NP is best explained as episodic retrieval, wherein retrieval is governed by a host of primeprobe contextual relationships. Those contextual factors can involve memory for temporal information (Neill, 1997; Neill et al., 1992), the prime (Malley \& Strayer, 1995) and probe components (Tipper et al., 1991), the predictability of probes from primes (Lowe, 1979), and the blend of materials composing the prime and probe sets (Lowe, 1979; Moore, 1994).

Context dependency of NP could be theoretically important. That compatibility between sequential environments governs the occurrence of NP supports the claim that NP has adaptive consequences for future processing. Tipper et al. (1991) noted that, in most situations, particular stimuli are consistently relevant and are selected for response, whereas other stimuli are consistently irrelevant and are 
ignored. Consequently, when the same environments are encountered, the same stimuli are again categorized as relevant or irrelevant and are attended or ignored, respectively. "Therefore, if the prior experience of ignoring a stimulus [in the same environment] can be reinstated, then selective processing will be facilitated" (Tipper et al., 1991, p. 691). Facilitated processing for ATT items, where across environments, targets are consistently relevant and distractors are consistently irrelevant, supports this claim. Similarly, when that prior experience of "ignoring" yields performance impairments, as during inconsistent IGN trials, "the very mechanism by which attention to a particular source of information can be maintained effectively over time" (Neill et al., 1992, p. 999) may be revealed. From this perspective, sustained attention to some information is an effect (Johnson \& Dark, 1986) when current processing is guided by the constraints, or results, of memory for past processing in that situation.

When NP depends on reinstatement of a prior experience of ignoring a stimulus, how does context work to yield opposite PP and NP effects? The answer depends on achieving an understanding of what has "gotten into" the representations of the particular processing episodes stored during practice, and what is later "taken out" of them during test (Logan \& Etherton, 1994, p. 1022). A satisfactory explanation also requires an appreciation of what subjects learn and remember about the general environment (Chawarski \& Sternberg, 1993), or "list-wide" context (Smith et al., 1989) when those episodic memories are acquired.

Logan and Etherton (1994) consider that instances of processing occasions represent co-occurrences, or particular combinations of the goal, stimuli encountered, interpretations given to the stimuli, and response made. They found that when word pairs were consistently repeated, subjects learned and remembered which words went together, forming stimulus-stimulus (S-S) connections, and the responses made to the pairs, forming stimulus-response (S-R) connections. They showed that RTs improved with practice, indicating that performance relied on memory for regular co-occurrences of word pairings. When transferred to a new environment in which word pairings changed, subjects were forced to find new bases to support performance. The search for an alternate platform was revealed by slowed RTs and quantitatively changed performances.

By applying these notions, one can characterize how the particular co-occurrences that were represented during practice changed during test: (1) Across all experiments, during IGN trials, established S-R connections became unreliable; (2) when distractors were paired with novel words during IGN probes, the S-S connections were broken; (3) "flipped" IGN probes maintained the established S-S connections; (4) when test involved new items, correct responses were not available from memory and were provided by the algorithm; and (5) when there were no new pairs, all test responses had some type of episodic representation.

Within this framework, the opposite PP and NP effects for distractors in Experiments 1 and 3 are, perhaps, easiest to understand. Retrieval was determined by the effec- tiveness of the different probes in cuing earlier experiences of ignoring specific words. Those experiences were better reinstated by the intact $\mathrm{S}-\mathrm{S}$ associations provided by "flipped" probes because the specific context in which the distractors were ignored was repeated (Tulving \& Thomson, 1973). Performance was impaired because memory that specific words had been consistently goal irrelevant and should be ignored was retrieved, thereby interfering with current responding. In contrast, those earlier experiences were not reinstated by the broken S-S connections provided by changed IGN probes in Experiment 1, and there was no effect of memory that specitic words had been ignored.

Changes in general context also influenced memory access. Targets and distractors became connected to the general background in which those items were differently processed. General context affected memory access during test, for what was retrieved depended on whether or not the background was reinstated. In both Experiments 1 and 3, unfamiliar, or new, items were part of test. Nonetheless, in Experiment 3, since two thirds of all the trials involved familiar S-S connections, practice and test lists were mostly related. Memory for co-occurrences during earlier processing (Logan \& Etherton, 1994) was retrieved. Information that specific words had been ignored and should continue to be ignored was retrieved, yielding NP. In contrast, in Experiment 1, only one third of test trials involved familiar S-S connections, and practice and test lists were mostly unrelated. Thus, the context associated with ignoring items during practice was not reinstated during test. Consequently, there were no retrieval cues for information about what was done with an item during practice. Memories of past processing during which specific words had been ignored were not retrieved, and NP was not obtained.

Beyond implications for theories of cognition that conjoin processes of attention and memory, the present results indicate that context, by itself, produces degrees of relatedness that are sufficient to produce qualitatively different priming, or transfer, phenomena. These findings have important implications for choosing suitable baselines against which transfer across processing tasks and environments should be assessed. That inclusion or exclusion of particular baseline events can determine type of transfer is worthy of further investigation.

\section{REFERENCES}

BAdDeley, A. D. (1976). The psychology of memory. New York: Basic Books.

BatTIG, W. F., \& ToGGLiA, M. P. (1978). Handbook of semantic word norms. Hillsdale, NJ: Erlbaum.

ChaWARski, M. C., \& S Ternberg, R. J. (1993). Negative priming in word recognition: A context effect. Journal of Experimental Psychology: General, 122, 195-206.

Dalrymple-Alford, E. C., \& Budayr, B. (1966). Examination of some aspects of the Stroop color-word test. Perceptual \& Motor Skills, 23, 1211-1214.

DeSchePper, B., \& Treisman, A. [M.] (1991, November). Novel visual shapes in negative priming. Paper presented at the meeting of the Psychonomic Society, San Francisco.

DeSchepper, B., \& Treisman, A. [M.] (1996). Visual memory for novel 
shapes: Implicit coding without attention. Journal of Experimental Psychology: Learning, Memory, \& Cognition, 22, $27-47$.

Fox, E. (1995). Negative priming from ignored distractors in visual selection: A review. Psychonomic Bulletin \& Review, 2, 145-173.

Hasher, L., Zacks, R. T., Stolzfus, E. R., Kane, M. J., \& Connelly, S. L. (1996). On the time course of negative priming: Another look. Psychonomic Bulletin \& Review, 3, 231-237.

Houghton, G., \& TipPER, S. P. (1994). A model of inhibitory mechanisms in selective attention. In D. Dagenbach \& T. H. Carr (Eds.), Inhibitory processes in attention, memory, and language (pp. 53-112). San Diego: Academic Press.

Johnson, J. A., \& DARK, V. J. (1986). Selective attention. Annual Review of Psychology, 37, 43-75.

LOGAN, G. D. (1988). Toward an instance theory of automatization. Psychological Review, 95, 492-527.

LogAN, G. D., \& Etherton, J. L. (1994). What is learned during automatization? The role of attention in constructing an instance. Journal of Experimental Psychology: Learning, Memory, \& Cognition, 20 1022-1050.

LowE, D. G. (1979). Strategies, context, and the mechanism of response inhibition. Memory \& Cognition, 7, 382-389.

MALleY, G. B., \& STRAYER, D. L. (1995). Effect of stimulus repetition on positive and negative identity priming. Perception \& Psychophysics, 57, 657-667.

MaY, C. P., Kane, M. J., \& HaSher, L. (1995). Determinants of negative priming. Psychological Bulletin, 118, 35-54.

MilLIKEN, B., \& JOORDENS, S. (1996). Negative priming without overt prime selection. Canadian Journal of Experimental Psychology, 50, 333-346.

Milliken, B., Joordens, S., Merikle, P., \& Seiffert, A. (1995). Selective attention: A reevaluation of the implications of negative priming. Manuscript submitted for publication.

MOORE, C. M. (1994). Negative priming depends on probe-trial conflict: Where has all the inhibition gone? Perception \& Psychophysics, 56 , 133-147.

NeEly, J. H., \& DurgonoĞLu, A. Y. (1985). Dissociative episodic and semantic priming effects in episodic recognition and lexical decision tasks. Journal of Memory \& Language, 24, 466-489.

NeILL, W. T. (1977). Inhibitory and facilitatory processes in attention. Journal of Experimental Psychology: Human Perception \& Performance, 3, 444-450.

NEILL, W. T. (1997). Episodic retrieval in negative priming and repeti- tion priming. Journal of Experimental Psychology: Learning, Memory, \& Cognition, 23, 1291-1305.

Neill, W. T., \& Valdes, L. A. (1992). Persistence of negative priming: Steady-state or decay? Journal of Experimental Psychology: Learning Memory, \& Cognition, 18, 565-576.

Neill, W. T., Valdes, L. A., \& TerRY, K. M. (1995). Selective attention and the inhibitory control of cognition. In F. N. Dempster \& C. J. Brainerd (Eds.), Interference and inhibition in cognition (pp. 207-261). San Diego: Academic Press.

Neill, W. T., Valdes, L. A., Terry, K. M., \& Gorfein, D. S. (1992) Persistence of negative priming: II. Evidence for episodic trace retrieval. Journal of Experimental Psychology: Learning, Memory, \& Cognition, 18, 993-1000.

Neumann, E., \& DeSchepper, B. G. (1991). Costs and benefits of target activation and distractor inhibition in selective attention. Journal of Experimental Psychology: Learning, Memory, \& Cognition, 17, 1136-1145.

Neumann, E, \& DeSchepper, B. G. (1992). An inhibition-based fan effect: Evidence for an active suppression mechanism in selective attention. Canadian Journal of Psychology, 46, 11-50.

Smith, M. C., Macleod, C. M., Bain, J. D., \& Hoppe, R. B. (1989). Lexical decision as an indirect test of memory: Repetition and list-wide priming as a function of type of encoding. Journal of Experimental Psychology: Learning, Memory, \& Cognition, 15, 1109-1118.

STROOP, J. R. (1935). Studies of interference in serial verbal reactions. Journal of Experimental Psychology, 18, 643-662.

TIPPER, S. P. (1985). The negative priming effect: Inhibitory priming by ignored objects. Quarterly Journal of Experimental Psychology, 37A, $571-590$

Trpper, S. P., \& Cranston, M. (1985). Selective attention and priming: Inhibitory and facilitatory effects of ignored primes. Quarterly Journal of Experimental Psychology, 37A, 591-611.

Tipper, S. P., Weaver, B., Cameron, S., Brehaut, J. C., \& Bastedo, J. (1991). Inhibitory mechanisms of attention in identification and localization tasks: Time course and disnuption. Journal of Experimental Psychology: Learning, Memory, \& Cognition, 17, 681-692.

TuLVING, E., \& THOMSON, D. M. (1973). Encoding specificity and retrieval processes in episodic memory. Psychological Review, 80, 352-373.

(Manuscript received April 19, 1996; revision accepted for publication March 28, 1997. 